\title{
Reporting and evaluating wait times for urgent hip fracture surgery in Ontario, Canada
}

\author{
Daniel Pincus MD, David Wasserstein MD MPH, Bheeshma Ravi MD PhD, James P. Byrne MD, Anjie Huang MSc, \\ J. Michael Paterson MSc, Avery B. Nathens MD PhD, Hans J. Kreder MD MPH, Richard J. Jenkinson MD MSc, \\ Walter P. Wodchis PhD
}

Cite as: CMAJ 2018 June 11;190:E702-9. doi: 10.1503/cmaj.170830

\begin{abstract}
BACKGROUND: Although a delay of 24 hours for hip fracture repair is associated with medical complications and costs, it is unknown how long patients wait for surgery for hip fracture. We describe novel methods for measuring exact urgent and emergent surgical wait times (in hours) and the factors that influence them.
\end{abstract}

METHODS: Adults aged 45 years and older who underwent surgery for hip fracture (the most common urgently performed procedure) in Ontario, Canada, between 2009 and 2014 were eligible. Validated data from linked health administrative databases were used. The primary outcome was the time elapsed from hospital arrival recorded in the National Ambulatory Care Reporting System until the time of surgery recorded in the Discharge Abstract Database (in hours). The influence of patient, physician and hospital factors on wait times was investigated using 3-level, hierarchical linear regression models.

RESULTS: Among 42230 patients with hip fracture, the mean (SD) wait time for surgery was 38.76 (28.84) hours, and 14174 (33.5\%) patients underwent surgery within 24 hours. Variables strongly associated with delay included time for hospital transfer (adjusted increase of $26.23 \mathrm{~h}, 95 \% \mathrm{Cl} 25.38$ to 27.01 ) and time for preoperative echocardiography (adjusted increase of $18.56 \mathrm{~h}, 95 \% \mathrm{Cl}$ 17.73 to 19.38$)$. More than half of the hospitals (37 of $72,51.4 \%$ ), compared with $4.8 \%$ of surgeons and $0.2 \%$ of anesthesiologists, showed significant differences in the risk-adjusted likelihood of delayed surgery.

INTERPRETATION: Exact wait times for urgent and emergent surgery can be measured using Canada's administrative data. Only one-third of patients received surgery within the safe time frame ( $24 \mathrm{~h})$. Wait times varied according to hospital and physician factors; however, hospital factors had a larger impact.
$\mathrm{H}$ ip fracture repair that is delayed more than 24 hours after hospital presentation is associated with increased medical complications ${ }^{1}$ and health care costs. ${ }^{2,3}$ Despite known consequences for delays, it is unknown how long patients wait for hip fracture repair and other urgent and emergent procedures across Canada. ${ }^{4}$ Studies about urgent and emergent surgical wait times have been conducted at single centres, ${ }^{3,5-10}$ and time was measured imprecisely. ${ }^{11-14}$ Although variables capturing exact wait times from hospital arrival were introduced to Canadian hospital discharge abstracts in 2009, studies have not used these data to describe and evaluate wait times for urgent surgery. ${ }^{1,14,15}$

We investigated these new time-to-surgery data among a population-based cohort of patients requiring surgery for hip fracture, the most common urgently performed surgical procedure in Canada. ${ }^{16}$ Our objectives were to use these data to measure wait times for surgery for hip fracture, identify modifiable factors influencing them, and determine whether variation is due to treatment by different hospitals or physicians, or both.

\section{Methods}

\section{Data sources and setting}

We conducted a population-based cross-sectional cohort study of patients with hip fractures who were treated in Ontario. Data were obtained from several administrative databases linked at the Institute for Clinical Evaluative Sciences (ICES, www.ices.on.ca). These databases have been used previously to study patients with hip fracture, ${ }^{12,17-19}$ in which sensitivity and positive predictive values for diagnosis of hip fracture are 95\% (Appendix 1A, available at www. cmaj.ca/lookup/suppl/doi:10.1503/cmaj.170830/-/DC1). ${ }^{20}$ We chose to study hip fractures because surgery for hip fracture is the most common urgently performed procedure in Canada, ${ }^{16}$ and wait times are already used as quality-of-care indicators worldwide..$^{21-23}$

\section{Participants}

We considered adults aged 45 years and older who underwent surgery for hip fracture in Ontario from Apr. 1, 2009, through 
Mar. 31, 2014, to be eligible. Accrual began when exact surgery start times were introduced in the databases utilized, enabling us to calculate precise wait times for each patient (in hours) in the cohort. ${ }^{15}$ We excluded patients aged 45 years and younger, as well as others unrepresentative of patients with osteoporotic hip fractures, consistent with prior ${ }^{1,2}$ and ongoing ${ }^{24}$ hip fracture research (Supplementary Table 1, Appendix 1B, contains the full list of exclusion criteria).

\section{Outcome measure}

The primary dependent variable was the total time elapsed (in hours) between arrival at the emergency department (at the first hospital, if interfacility transfer occurred ${ }^{25}$ recorded in the National Ambulatory Care Reporting System and surgery for hip fracture recorded in the Discharge Abstract Database. We assessed the relative contribution of specific phases of care to observed wait times by calculating separately the time spent in the emergency department, during hospital transfer and after hospital admission.

For each patient, we recorded acute conditions that may benefit from medical treatment (and delay) before surgery. Specific conditions were taken from the National Institute for Health and Care Excellence 124 guideline. ${ }^{26}$

We assessed several characteristics previously shown to influence surgical delays in other single-centre studies, including age, sex and medical comorbidity. ${ }^{3,5-10}$ Characteristics determined after surgery were not considered, even if these factors were surrogates for patient case mix, such as surgery duration, discharge disposition or length of stay. ${ }^{27}$ Comorbidities listed on hospital discharge abstracts in the 5 years before the patient's hip fracture were categorized according to the Deyo-Charlson Comorbidity Index. ${ }^{28}$ Previously validated algorithms identified frail patients ${ }^{29}$ and those with diabetes, ${ }^{30}$ hypertension, ${ }^{31}$ chronic obstructive pulmonary disease, ${ }^{32}$ congestive heart failure, coronary artery disease ${ }^{33}$ or polytrauma (defined as an Injury Severity Score $\geq 16$ ) at the time of their injury. We used median neighbourhood household income quintiles as a proxy for socioeconomic status, ${ }^{34-36}$ and we identified patients residing in rural areas using the Rurality Index of Ontario. ${ }^{36}$ We also considered antiplatelet and anticoagulant prescriptions dispensed to patients within 1 year before surgery for those with Ontario Drug Benefit coverage (i.e., all those $>65$ years of age). ${ }^{37}$ Each fracture and procedure type were recorded.

We assessed and assigned physician- and hospital-related factors at the time of each patient's operation. These included years since each surgeon's Canadian orthopedic certification ("surgeon experience") and the number of hip fracture procedures performed in the year preceding the index event ("surgeon and hospital volume”). Each hospital's capacity for performing nonelective surgery was operationalized as the average daily number of any nonelective (or "urgent") procedures performed at the hospital, orthopedic or otherwise, in the year preceding the index event. Hospitals were also categorized as being either "academic," "large community" or "small/medium community" (> 400 or $<400$ beds, respectively)..$^{38}$ We identified patients directly transferred from other hospitals and other health care institutions (e.g., long-term care) by standard protocols. ${ }^{25}$ The time of hospital arrival was categorized as "working hours" 8 am-4 pm, "evening" 4 pm-12 pm, or "overnight" 12 midnight-8 am, and "weekend" or "weekday." We also described the proportion of surgical procedures occurring overnight (12 midnight-8 am) using surgeon billing codes. ${ }^{19,39}$ Finally, we recorded preoperative internal medicine consultations, anesthesia consultations and echocardiograms that occurred between hospital arrival and the time of surgery.

\section{Statistical analysis}

We used simple (single-level) linear regression to relate the above predictors ("potential factors influencing wait times for surgery") to surgical wait times, analyzed as a continuous variable in hours. ${ }^{40-42}$ We used standardized $\beta$ coefficients with $95 \%$ confidence intervals (Cls) to report increases and decreases in wait times (in hours) associated with each predictor variable. We also used 3-level hierarchical linear regression models to explore the relative contribution of physician and hospital factors to variation in wait times. The random-effects output from this model provided each physician's and hospital's unique adjusted wait time difference compared with the cohort average (i.e., increase or decrease in adjusted wait time [in hours] and 95\% Cl). We performed the physician-level analysis twice, considering surgeons and anesthesiologists in separate models that were crossclassified to account for physicians working at more than 1 hospital (Appendix 1C). ${ }^{43,44}$

To quantify the relative effect of individual physicians and hospitals on variability in wait times, we measured the proportion of physicians and hospitals that were "outliers" compared with their peers. "Low outliers" - physicians and hospitals with wait times significantly lower than average - were those with upper limits of the $95 \% \mathrm{Cl}$ wait time less than 0. Conversely, "high outliers" - physicians and hospitals with wait times that were significantly longer than average - were those with lower limits of their $95 \% \mathrm{Cl}$ wait time greater than $0.45,46$ To validate the effect of individual physicians and hospitals on variability in wait times, we reran the 3-level hierarchical regression model with clinical outcomes (30-d mortality, surgical complications) and medical costs in place of wait times (Appendix 1D).

All analyses were performed on linked, coded data at the Institute for Clinical Evaluative Sciences using SAS software (SAS version 9.3, SAS Institute), and we set type I error probability to 0.05 . We excluded patients with missing data $(<1 \%$ for all variables considered [Table 1]) from the regression models.

\section{Ethics approval}

The study protocol was approved by the Research Ethics Board at Sunnybrook Health Sciences Centre, Toronto.

\section{Results}

We included 42230 patients in our study. These patients were treated by 522 surgeons and 963 anesthesiologists from 72 hospitals. Patient mean (standard deviation [SD]) age was 80.77 (SD 10.67) years and most were female ( $n=29759,70.5 \%)$. Mean wait time for surgery after arrival at the emergency 
department was 38.76 (SD 28.84) hours. Mean time spent in the emergency department was 7.58 (SD 11.87) hours. Almost half of all patients received a preoperative internal medicine consultation ( $n=20781,49.2 \%), 11410$ (27.0\%) received a preoperative anesthesia consultation, and 2354 (5.6\%) underwent an echocardiogram before surgery. Nearly 1 in 5 patients older than 65 years (18.9\%) were prescribed antiplatelet or anticoagulant medications within a year before their hip fracture. About $9 \%(n=4136)$ of patients presented for surgery with an acute condition that may have benefitted from medical treatment (and delay) before surgery. Other characteristics of the cohort are displayed in Table 1.

Although most patients (> 75\%) were admitted to hospital within 6 hours of presentation at the emergency department

\section{Table 1 (part 1 of 1): Baseline characteristics of patients}

undergoing surgery for hip fracture in Ontario between 2009 and 2014

\begin{tabular}{|c|c|}
\hline Characteristic & $\begin{array}{c}\text { No. }(\%)^{\star} \text { of } \\
\text { patients } \\
n=42230\end{array}$ \\
\hline \multicolumn{2}{|l|}{ Wait times } \\
\hline Time from presentation to surgery, mean $\pm \mathrm{SD} ; \mathrm{h}$ & $38.76 \pm 28.84$ \\
\hline Time spent in the emergency department, mean $\pm \mathrm{SD}$; $\mathrm{h}$ & $7.58 \pm 11.87$ \\
\hline Time from admission to surgery, mean $\pm \mathrm{SD} ; \mathrm{h}$ & $31.18 \pm 26.54$ \\
\hline $\begin{array}{l}\text { Surgery conducted }>48 \mathrm{~h} \text { after presentation at the emergency } \\
\text { department }\end{array}$ & $11088(26.3)$ \\
\hline \multicolumn{2}{|l|}{ Patients } \\
\hline Age, mean $\pm S D$; yr & $80.77 \pm 10.67$ \\
\hline Female sex & $29759(70.5)$ \\
\hline Rural residence & $3611(8.6)$ \\
\hline \multicolumn{2}{|l|}{ Income quintile } \\
\hline 1 (lowest) & $9503(22.5)$ \\
\hline 2 & $8592(20.3)$ \\
\hline 3 & $7991(18.9)$ \\
\hline 4 & $8086(19.1)$ \\
\hline 5 (highest) & $7853(18.6)$ \\
\hline Missing data & $285(0.7)$ \\
\hline \multicolumn{2}{|l|}{ Medical comorbidity } \\
\hline \multicolumn{2}{|l|}{ Deyo-Charlson Comorbidity Index score } \\
\hline 0 & $6812(16.1)$ \\
\hline 1 & $5966(14.1)$ \\
\hline 2 & $3785(9.0)$ \\
\hline$\geq 3$ & $6223(14.7)$ \\
\hline No admission to hospital within the previous $5 \mathrm{yr}$ & $19444(46.0)$ \\
\hline Frailty & $7220(17.1)$ \\
\hline Diabetes & $12457(29.5)$ \\
\hline Hypertension & $33304(78.9)$ \\
\hline Chronic obstructive pulmonary disease & $8139(19.3)$ \\
\hline Coronary artery disease & $3119(7.4)$ \\
\hline Congestive heart failure & $9567(22.7)$ \\
\hline Institutionalized before admission & $12432(29.4)$ \\
\hline Antiplatelet or anticoagulant prescription $\dagger$ & $7977(18.9)$ \\
\hline \multicolumn{2}{|l|}{ Injury characteristics } \\
\hline Polytrauma (ISS $\geq 16$ ) & $329(0.8)$ \\
\hline \multicolumn{2}{|l|}{ Fracture type } \\
\hline Femoral neck & $21208(50.2)$ \\
\hline Intertrochanteric & $18544(43.9)$ \\
\hline Subtrochanteric & $2478(5.9)$ \\
\hline
\end{tabular}

(mean $7.58 \mathrm{~h}$ [SD 11.87]), only 14174 (33.5\%) received surgery within the recommended time frame (24 h) (Figure 1). 1,47,48 The proportion of patients with hip fracture is also reported by their time of presentation, admission and surgery in Appendix 2, available at www.cmaj.ca/lookup/suppl/doi:10.1503/cmaj.170830/-/ DC1. Whereas 5837 patients (13.8\%) arrived overnight, only 441 patients $(1.0 \%)$ received surgery during this time.

Results of the linear regression model relating potential risk factors to delayed surgery are shown in Table 2. Patient transfer for surgery was associated with more than 1 day of additional delay for surgery (adjusted increase of $26.23 \mathrm{~h}, 95 \% \mathrm{Cl} 25.38$ to 27.01). Preoperative consultations by internal medicine (adjusted increase of $6.43 \mathrm{~h}, 95 \% \mathrm{Cl} 6.06$ to 6.80 ) and anesthesia

Table 1 (part 2 of 2): Baseline characteristics of patients undergoing surgery for hip fracture in Ontario between 2009 and 2014

\section{Characteristic}

No. $(\%)^{*}$ of patients $n=42230$

\section{Physicians}

Surgeon

No. of cases of hip fracture seen in previous yr, mean \pm SD

$40.00 \pm 22.17$

Years since Canadian orthopedic certification, mean \pm SD

$12.60 \pm 9.63$

Preoperative workup

Preoperative consultation with internal medicine

$20781(49.2)$

Preoperative consultation with anesthesia

$11410(27.0)$

Preoperative echocardiogram

$2354(5.6)$

Type of surgery conducted

Sliding hip screw or cannulated screws

$19116(45.3)$

Arthroplasty

16248 (38.5)

Intramedullary nail

$6866(16.3)$

\section{System}

Hospital

Patient transferred from another facility

$2467(5.8)$

No. of cases of hip fracture in previous yr, mean \pm SD

No. of urgent surgeries per $d$, mean \pm SD

$50.22 \pm 112.30$

Type

Academic

Large community

Medium community

Missing data

$4.52 \pm 2.61$

12167 (28.8)

$15940(37.7)$

13741 (32.5)

$382(0.9)$

Timing of patient presentation

Weekend

12136 (28.7)

Evening

Working hours

16426 (38.9)

19967 (47.3)

Overnight

$5837(13.8)$

Surgery conducted overnight

$441(1.0)$

Year surgery conducted

2009

$7848(18.6)$

2010

$8139(19.3)$

$8295(19.6)$

2011

$8643(20.5)$

2013

Note: ISS = Injury Severity Score, SD = standard deviation

*Unless specified otherwise.

†Data for antiplatelet or anticoagulant prescriptions were available and reported only for patients aged 65 years and older. 
(adjusted increase of $5.90 \mathrm{~h}, 95 \% \mathrm{Cl} 5.48$ to 6.33 ), as well as preoperative echocardiography (adjusted increase of $18.56 \mathrm{~h}$, $95 \% \mathrm{Cl} 17.73$ to 19.38 ) were also associated with significant delays after adjustment.

Results of our hierarchical linear regression models are shown in Figures 2A-C. More than half of the hospitals (37 of 72, 51.4\%) showed significant differences in the likelihood of delays in surgery for hip fracture that were not attributable to patient case mix and physician random effects (Figure $2 \mathrm{~A}$ ). Conversely, only 25 of $522(4.8 \%)$ surgeons and 2 of $963(0.2 \%)$ anesthesiologists were outliers or significantly different in their likelihood of performing delayed surgery after adjustment for patient and hospital factors. Similarly, adjusted odds of mortality, surgical complications and medical costs varied between hospitals (9.7\%, 16.7\% and $38.8 \%$ were outliers for each outcome, respectively) but not between physicians (no surgeons and anesthesiologists were significantly different for these outcomes) (Appendix 1D).

\section{Interpretation}

Wait times varied significantly depending on where patients were treated, with more than half of hospitals (51.4\%) showing significant differences in the likelihood of delayed surgery for hip fracture that was not attributable to patient or physician factors. Transfers, preoperative consultations, echocardiography and prescriptions for anticoagulants are important and modifiable causes of delay. Two-thirds (66\%) of the participants did not receive surgery within the safe time frame $(24$ h). 1,47,48 Variation within Ontario's public health care system warrants performance improvement at the hospital level.

Variation in wait times was attributable to treatment at different hospitals, as opposed to treatment by different physicians. As such, initiatives for quality improvement may target hospitallevel processes preferentially rather than individual physician practices. In contrast, and contrary to calls for physician-level reporting,,$^{50-52}$ the finding that wait times, clinical outcomes and costs were similar between physicians after accounting for patient and hospital factors suggests such reporting may be less informative than hospital-level information. Examples of hospital-level interventions include medical and surgical comanagement models, ${ }^{53}$ and policies for preoperative consultations, echocardiography and anticoagulant reversal, ${ }^{10}$ which may ensure that coordinated care does not compromise the provision of timely surgery. Policies between hospitals should also address patients who require transfer for surgery, balancing the risk of treatment at smaller centres ${ }^{38}$ with delays associated with these transfers, both of which are known risk factors for mortality. ${ }^{38,54,55}$ A successful surgical coverage algorithm in this regard was developed in Manitoba, where rural hospitals were matched to surgical hospitals that agreed to accept patients from rural areas regardless of bed availability. ${ }^{10}$ Other solutions may be to designate "urgent surgical centres" ${ }^{56}$ with

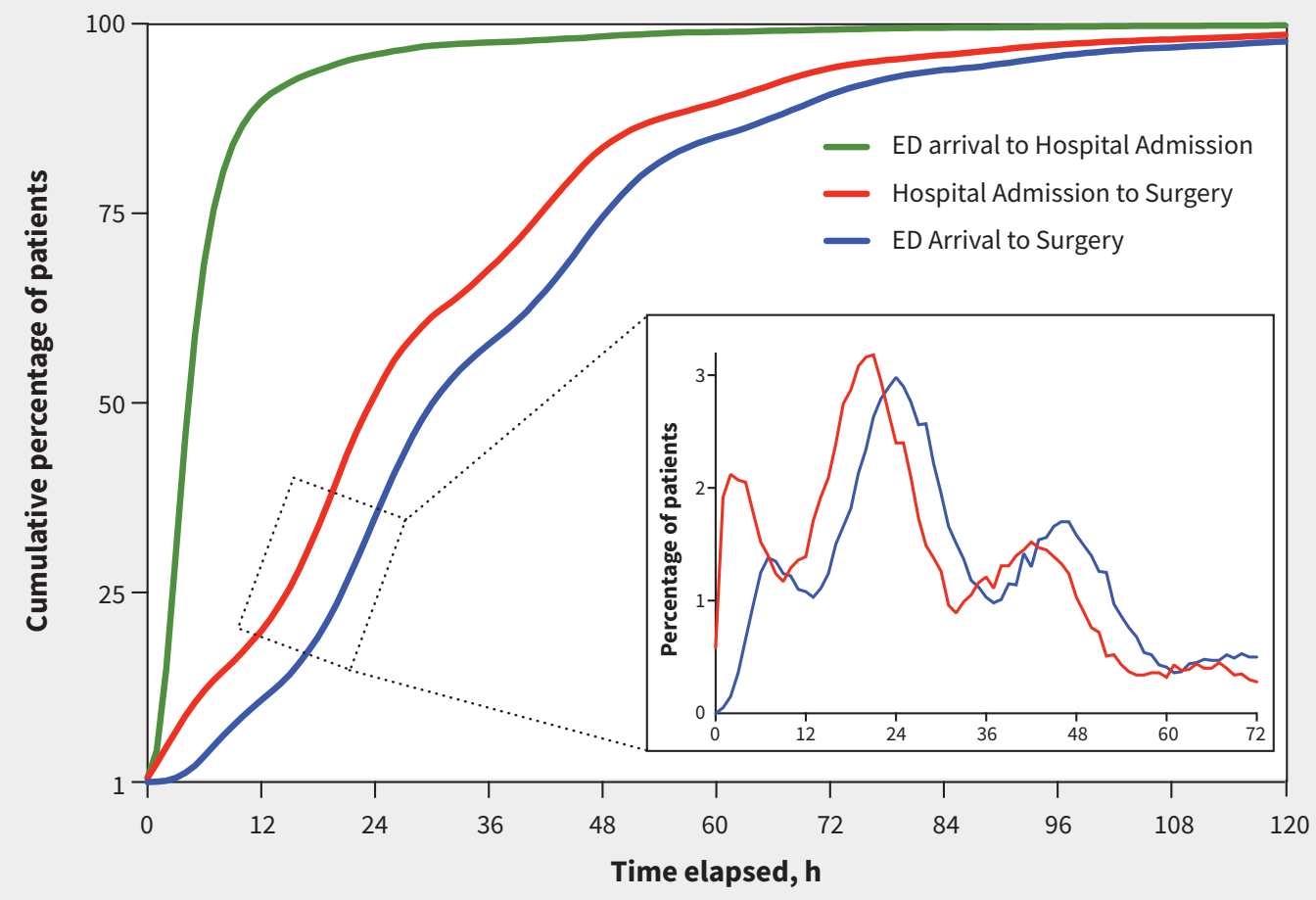

Figure 1: Cumulative percentage of patients with hip fracture by time elapsed (in h) from arrival at the emergency department (ED) to hospital admission (green line), hospital admission to undergoing surgery (red line) and arrival at the ED to undergoing surgery (blue line). One-third of patients ( $n=$ $14174,33.5 \%)$ underwent surgery within the safe time frame $(24 \mathrm{~h})$. The inset-shows the exact proportion of patients receiving surgery by the time elapsed, illustrating that wait times for surgery for hip fracture follow a sinusoidal distribution. 
Table 2: Linear regression model relating potential risk factors to wait times for surgery (modelled as a continuous variable)*

Adjusted increase or decrease in wait time $(95 \% \mathrm{Cl}), \mathrm{h}$

\section{Patient characteristic}

Age, per yr

0.042 (0.023 to 0.061 )

Female sex

Rural residence

Income quintile ( 1 [lowest] v. 5 [highest])

Income quintile ( 2 v. 5 )

Income quintile ( 3 v. 5 )

Income quintile (4 v. 5)

$-1.11(-1.50$ to -0.710$)$

$-0.200(-0.874$ to 0.476$)$

$0.041(-0.511$ to 0.592$)$

$-0.182(-0.746$ to 0.382$)$

$-0.032(-0.604$ to 0.540$)$

$0.364(-0.206$ to 0.934$)$

Medical comorbidity

Charlson group (v. no previous hospital admissions)

0

1

2

3

Frailty

Diabetes

Hypertension

Chronic obstructive pulmonary disease

Coronary artery disease

Congestive heart failure

Preadmission institutionalization

Antiplatelet or anticoagulant prescriptiont

Injury characteristics

Polytrauma (ISS $\geq 16$ )

Subtrochanteric fracture (v. femoral neck)

Intertrochanteric fracture ( $v$. femoral neck)

\section{Physician characteristics}

\section{Surgeon}

No. of cases of hip fracture in previous yr

Years since Canadian orthopedic certification

Preoperative workup

Preoperative consultation with internal medicine

Preoperative consultation with anesthesia

Preoperative echocardiogram

1.01 (0.494 to 1.528$)$

$1.13(0.553$ to 1.704$)$

1.438 ( 0.752 to 2.125$)$

2.19 ( 1.55 to 2.83 )

$0.368(-0.162$ to 0.900$)$

$0.081(-0.331$ to 0.494$)$

0.501 ( 0.037 to 0.966$)$

$0.140(-0.329$ to 0.609$)$

$0.628(-0.084$ to 1.34$)$

3.10 (2.63 to 3.57 )

$0.267(-0.157$ to 0.692$)$

5.80 (5.31 to 6.29 )

-1.67 (-3.72 to 0.353$)$

0.275 ( -0.653 to 1.20$)$

$0.335(-0.195$ to 0.866$)$

Type of surgery

Arthroplasty (v. intramedullary nail)

Sliding hip screw or cannulated screws (v. intramedullary nail)

$-0.025(-0.033$ to -0.017$)$

$-0.028(-0.047$ to -0.009$)$

$6.43(6.06$ to 6.80$)$

5.90 (5.48 to 6.33 )

18.56 (17.73 to 19.38$)$

$-1.68(-2.25$ to -1.12$)$

0.884 (0.154 to 1.61$)$

\section{System characteristics}

Hospital

Patient transferred from another facility

No. of cases of hip fracture in previous yr

Mean no. of urgent surgeries per d

Academic (v. medium community hospital)

Large (v. medium community hospital)

Timing of presentation

Admission over weekend

After-hours presentation (v. working hours)

Overnight presentation (v. working hours)

26.24 (25.38 to 27.09$)$

$-0.014(-0.017$ to -0.012$)$

$1.08(0.947$ to 1.21$)$

3.15 (2.52 to 3.78 )

4.20 (3.73 to 4.66 )

-2.71 ( -3.10 to -2.32$)$

$-1.68(-2.22$ to -1.14$)$

1.00 (0.621 to 1.38$)$

Year of surgery

2009 (v. 2013)

1.53 (0.970 to 2.09)

2.49 (1.94 to 3.05 )

1.42 (0.874 to 1.97 )

2011 (v. 2013)

2012 (v. 2013)

$0.265(-0.275$ to 0.805$)$

Note: $\mathrm{Cl}=$ confidence interval, ISS = Injury Severity Score

*We conducted the analysis for 40508 patients (without statistical outliers; see Appendix 1C, available at www.cmaj.ca/lookup/suppl/doi:10.1503/cmaj.170830/-/DC1).

We adjusted all regression models for the following covariates: age, sex, year of surgery, income quintile, Charlson group, frailty, diabetes, coronary artery disease,

chronic obstruction pulmonary disease, coronary artery disease, preadmission institutionalization, Injury Severity Score, and fracture and surgery type.

†Calculated in a separate linear regression model that was restricted to patients for whom prescription receipts were available (those aged $65 \mathrm{yr}$ or older). 
A

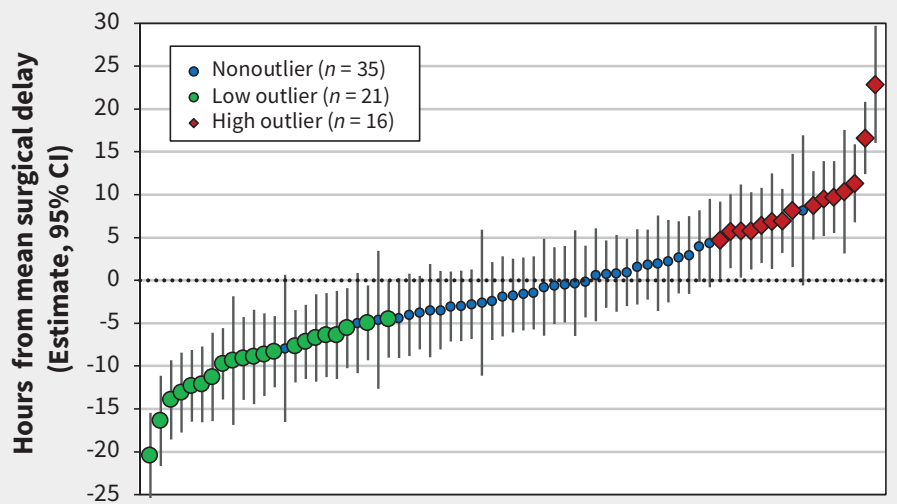

Hospital $n=72,51.4 \%$ outliers

B

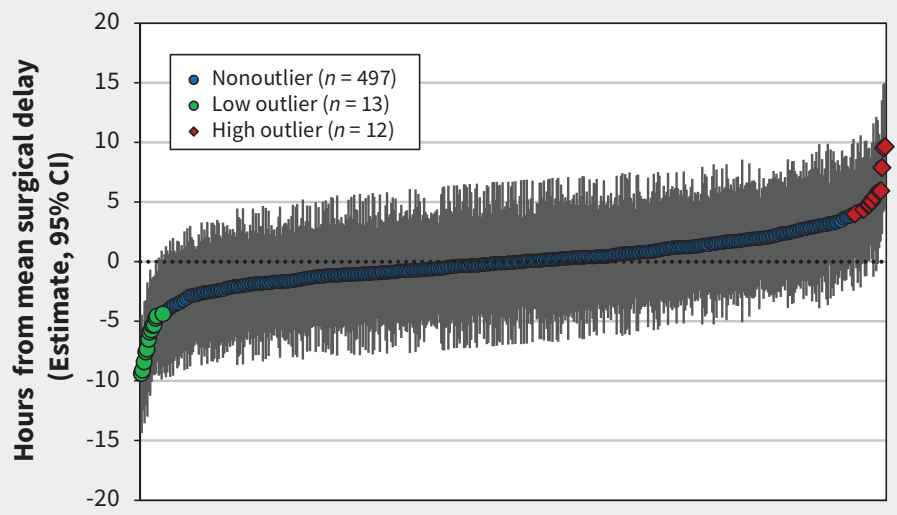

Surgeon $(n=522), 4.8 \%$ outliers

C

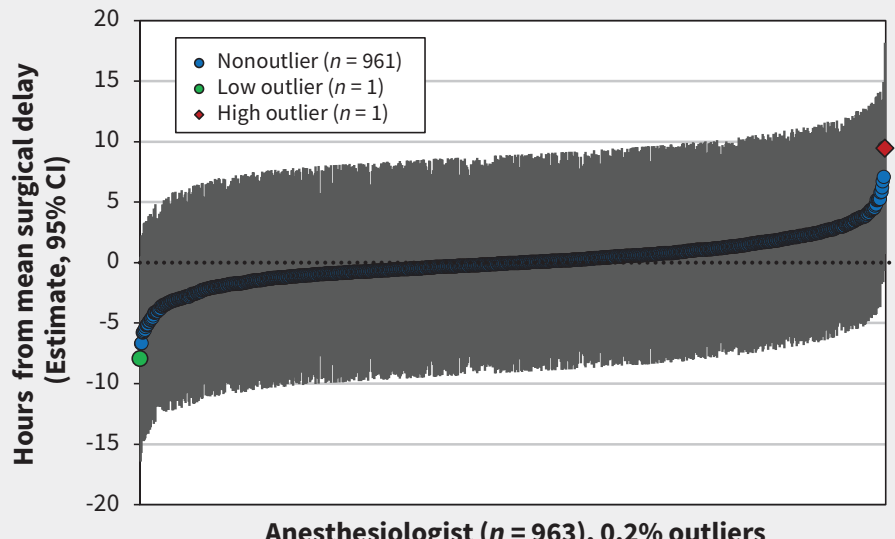

Figure 2: (A) Mean differences (in h, with 95\% confidence intervals [Cls]) for each hospital from the average surgical delay in the cohort was estimated in a 3-level linear regression model, adjusted for patient case mix and surgeon random effects. We classified hospitals that were significantly more likely to have early surgery performed as "low" outliers (green) and those that were significantly more likely to have delayed surgery performed as "high" outliers (red). More than half of the hospitals ( 37 of $72,51.4 \%$ ) showed significant differences in the likelihood of delayed surgery not attributable to patient case mix ( 1 hospital fell outside the graph area (estimate $=+90.1 \mathrm{~h}, 95 \% \mathrm{Cl} 77.2$ to 103.0 ). We conducted the analysis for 42025 patients (missing observations were excluded). (B) Mean differences (in $\mathrm{h}$, with $95 \% \mathrm{Cls}$ ) for each surgeon from the average surgical delay in the cohort was estimated in a 3-level linear regression model, adjusted for patient case mix and hospital random effects. We classified surgeons who were significantly more likely to perform early surgery as "low" outliers (green) and those who were significantly more likely to perform delayed surgery as "high" outliers (red). Only $4.8 \%$ of the surgeons (25 of 522 ) showed significant differences in the likelihood of delayed surgery not attributable to patient case mix or hospital random effects. We conducted the analysis for 42025 patients (missing observations were excluded). (C) Mean differences (in h, with $95 \% \mathrm{Cls}$ ) for each anesthesiologist from the average surgical delay in the cohort was estimated in a multilevel linear regression model, adjusted for patient case mix and hospital random effects. We classified anesthesiologists who were significantly more likely to enable early surgery as "low" outliers (green) and those who were significantly more likely to enable delayed surgery as "high" outliers (red). Only $0.2 \%$ of anesthesiologists (2 of 963) showed significant differences in the likelihood of delayed surgery not attributable to patient case mix or hospital random effects. We conducted the analysis for 11343 patients who had preoperative anesthesia consultations (missing observations were excluded). 
catchment areas large enough to sustain consistent daytime nonelective surgery volumes ${ }^{57}$ or to transfer patients to hospitals with available operating rooms.

Canadian surgeons may wait until after their elective procedures are completed before operating on urgent surgical patients. The finding that wait times for weekends were shorter is contrary to reports from other countries, and indirect evidence of this practice. That less than $5 \%$ of surgeons and less than $1 \%$ of anesthesiologists showed significant differences in delays is evidence that physicians may not be doing (or cannot do) enough to improve wait times for their patients. Policy that guarantees elective cases would be completed later in the day, even if nonelective cases are prioritized before them, may improve wait times for urgent procedures without the need to increase capacity in operating rooms.

We also found that reporting wait times from arrival at the emergency department is feasible because only $3 \%$ of patients were missing these data, and the time spent waiting in hospital transfer and the emergency department (mean $7.58 \mathrm{~h}$ [SD 11.87]) can be measured, which may provide another target for improved patient flow. An advantage of these Canadian data compared with data from the United States is the ability to capture exact wait times in hours (versus days) and the time elapsed in transfer between hospitals. ${ }^{48,58}$ Other potential applications of these data include more accurately identifying afterhours surgery, ${ }^{19}$ durations of surgery ${ }^{59}$ and overlapping surgical procedures. ${ }^{1,60,61}$

\section{Limitations}

Although specific reasons for delay could not be assessed in the data that were sampled, risk-adjusted differences observed between hospitals should not reflect clinical reasons, but rather processes of care at different hospitals. Furthermore, because only about $9 \%$ of patients presented with acute medical conditions that warranted delay, the scenario of rushing patients to surgery despite suspicious symptoms appears to be the exception rather than the rule. We have described new time variables that identify exact wait times (in hours) in Canada's administrative data. ${ }^{15}$ The variables have high face validity, including detecting differences when they were expected, such as longer delays among patients with comorbidity. ${ }^{5-10}$ Missing data for emergency department arrival times were uncommon ( $n=1460$ or $<3 \%$ ) and likely represented patients transferred from other health care institutions directly to inpatient beds. There were no missing data for surgery start times, which are used by Ontario's Surgical Efficiency Targets Program. ${ }^{49}$

\section{Conclusion}

Exact wait times for urgent and emergent surgery can be measured in Canada's administrative data. Only one-third of patients with hip fracture received surgery within the safe time frame (24 h). Because wait times vary according to where patients are treated, reporting and improvement efforts at the hospital level are required to ensure timely provision of urgent surgery for hip fracture. Reporting on physician performance, in contrast, may be less informative.

\section{References}

1. Pincus D, Ravi B, Wasserstein D, et al. Association between wait time and 30-day mortality in adults undergoing hip fracture surgery. JAMA 2017;318:1994-2003.

2. Pincus $D$, Wasserstein $D$, Ravi $B$, et al. Medical costs of delayed hip fracture surgery. J Bone Joint Surg. In press.

3. Mclsaac DI, Abdulla K, Yang H, et al. Association of delay of urgent or emergency surgery with mortality and use of health care resources: a propensity score-matched observational cohort study. CMAJ 2017;189:E905-12.

4. Sheehan KJ, Sobolev B, Villán Villán YF, et al. Patient and system factors of time to surgery after hip fracture: a scoping review. BMJ Open 2017;7:e016939.

5. Bergeron E, Lavoie A, Moore L, et al. Is the delay to surgery for isolated hip fracture predictive of outcome in efficient systems? J Trauma 2006;60:753-7.

6. Fantini MP, Fabbri G, Laus M, et al. Determinants of surgical delay for hip fracture. Surgeon 2011;9:130-4.

7. Ricci WM, Brandt A, McAndrew C, et al. Factors affecting delay to surgery and length of stay for patients with hip fracture. J Orthop Trauma 2015;29:e109-14.

8. Vidán MT, Sánchez D, Gracia Y, et al. Causes and effects of surgical delay in patients with hip fracture: a cohort study. Ann Intern Med 2011;155:226-33.

9. Charalambous CP, Yarwood S, Paschalides C, et al. Factors delaying surgical treatment of hip fractures in elderly patients. Ann R Coll Surg Engl 2003;85:117-9.

10. Bohm E, Loucks L, Wittmeier K, et al. Reduced time to surgery improves mortality and length of stay following hip fracture: results from an intervention study in a Canadian health authority. Can J Surg 2015;58:257-63.

11. Ryan DJ, Yoshihara H, Yoneoka D, et al. Delay in hip fracture surgery: an analysis of patient-specific and hospital-specific risk factors. J Orthop Trauma 2015;29:343-8.

12. Weller I, Wai EK, Jaglal S, et al. The effect of hospital type and surgical delay on mortality after surgery for hip fracture. J Bone Joint Surg Br 2005;87:361-6.

13. Neufeld ME, O'Hara NN, Zhan M, et al. Timing of hip fracture surgery and 30-day outcomes. Orthopedics 2016;39:361-8.

14. Born K, Bear R, Tierney R. Urgent surgery: A forgotten wait time? Healthy Debate [online]; 2013 Available: http://healthydebate.ca/2013/09/topic/wait -times-access-to-care/wait-times-for-urgent-surgery (accessed 2018 May 28).

15. Frood J, Johnson T. Improving measures of hip fracture wait times: a focus on Ontario. Healthc Q 2010;13:16.

16. Inpatient hospitalizations, surgeries, newborns and childbirth indicators, 20142015. Ottawa: Canadian Institute for Health Information; 2016. Available: https:// secure.cihi.ca/estore/productFamily.htm?locale=en\& (accessed 2018 May 28).

17. Nikitovic M, Wodchis WP, Krahn MD, et al. Direct health-care costs attributed to hip fractures among seniors: a matched cohort study. Osteoporos Int 2013; 24:659-69.

18. Pitzul KB, Wodchis WP, Carter MW, et al. Post-acute pathways among hip fracture patients: a system-level analysis. BMC Health Serv Res 2016;16:275.

19. Pincus DM, Desai SJ, Wasserstein DMMF, et al. Outcomes of after-hours hip fracture surgery. J Bone Joint Surg Am 2017;99:914-22.

20. Juurlink D, Preyra C, Croxford R, et al. Canadian Institute for Health Information Discharge Abstract Database: a validation study. Toronto (ON): Institute for Clinical Evaluative Sciences; 2006.

21. Wait times for priority procedures in Canada, 2017. Ottawa (ON): Canadian Institute for Health Information; 2017.

22. National Hip Fracture Database annual report 2015. London (UK): Royal College of Physicians; 2015.

23. ACS TQIP best practices in management of orthopaedic trauma. Chicago (IL): American College of Surgeons and Rosemount (IL): Orthopaedic Trauma Association; 2015. Available: www.facs.org/ /media/files/quality\%20programs/trauma/tqip/ tqip\%20bpgs\%20in\%20the\%20management $\% 20$ of\%20orthopaedic\%20traumafinal .ashx (accessed 2018 May 28).

24. Hip Fracture Accelerated Surgical Treatment and Care Track (HIP ATTACK) Investigators. Accelerated care versus standard care among patients with hip fracture: The HIP ATTACK pilot trial. CMAJ 2014;186:E52-60.

25. Canadian hospital reporting project technical notes - clinical indicators. Ottawa (ON): Canadian Institute for Health Information; 2013.

26. Guy P, Sheehan KJ, Morin SN, et al. Feasibility of using administrative data for identifying medical reasons to delay hip fracture surgery: a Canadian database study. BMJ Open 2017;7:e017869.

27. Hernán MA, Hernández-Díaz S, Robins JM. A structural approach to selection bias. Epidemiology 2004;15:615-25.

28. Deyo RA, Cherkin DC, Ciol MA. Adapting a clinical comorbidity index for use with ICD-9-CM administrative databases. J Clin Epidemiol 1992;45:613-9.

29. Weiner JP, Abrams C. The Johns Hopkins ACG ${ }^{\circledR}$ System: Technical Reference Guide Version 10.0; 2011.

30. Hux JE, Ivis F, Flintoft V, et al. Diabetes in Ontario: determination of prevalence and incidence using a validated administrative data algorithm. Diabetes Care 2002;25:512-6. 
31. Tu K, Campbell NR, Chen ZL, et al. Accuracy of administrative databases in identifying patients with hypertension. Open Med 2007;1:e18-26.

32. Gershon AS, Wang C, Guan J, et al. Identifying individuals with physcian diagnosed COPD in health administrative databases. COPD 2009;6:388-94.

33. Ko DT, Mamdani M, Alter DA. Lipid-lowering therapy with statins in high-risk elderly patients: the treatment-risk paradox. JAMA 2004;291:1864-70.

34. Agabiti N, Picciotto S, Cesaroni G, et al. The influence of socioeconomic status on utilization and outcomes of elective total hip replacement: a multicity populationbased longitudinal study. Int J Qual Health Care 2007;19:37-44.

35. Santaguida PL, Hawker GA, Hudak PL, et al. Patient characteristics affecting the prognosis of total hip and knee joint arthroplasty: a systematic review. Can J Surg 2008;51:428-36.

36. Kralj B. Measuring "rurality" for purposes of health care planning: an empirical measure for Ontario. Toronto (ON): Ontario Medical Association; 2005.

37. Pincus D, Gomes T, Hellings C, et al. A population-based assessment of the drug interaction between levothyroxine and warfarin. Clin Pharmacol Ther 2012;92:766-70.

38. Sheehan KJ, Sobolev B, Guy P, et al. In-hospital mortality after hip fracture by treatment setting. CMAJ 2016;188:1219-25.

39. Govindarajan A, Urbach DR, Kumar M, et al. Outcomes of daytime procedures performed by attending surgeons after night work. N Engl J Med 2015;373:845-53.

40. Ragland DR. Dichotomizing continuous outcome variables: dependence of the magnitude of association and statistical power on the cutpoint. Epidemiology 1992;3:434-40.

41. Bakhshi E, McArdle B, Mohammad K, et al. Let continuous outcome variables remain continuous. Comput Math Methods Med 2012;2012:639124.

42. Dawson NV, Weiss R. Dichotomizing continuous variables in statistical analysis: a practice to avoid. Med Decis Making 2012;32:225-6.

43. Goldstein H. Multilevel cross-classified models. Sociologic Methods Res 1994;22:364-75.

44. Browne W, Goldstein H, Rasbash J. Multiple membership multiple classification (MMMC) models. Stat Model 2001;1:103-24.

45. Shafi S, Nathens AB, Cryer HG, et al. The Trauma Quality Improvement Program of the American College of Surgeons Committee on Trauma. J Am Coll Surg 2009;209:521-30.e1.

46. Byrne JP, Xiong W, Gomez D, et al. Redefining "dead on arrival": identifying the unsalvageable patient for the purpose of performance improvement. J Trauma Acute Care Surg 2015;79:850-7.

47. Sayers A, Whitehouse MR, Berstock JR, et al. The association between the day of the week of milestones in the care pathway of patients with hip fracture and 30-day mortality: findings from a prospective national registry - The National Hip Fracture Database of England and Wales. BMC Med 2017;15:62.
48. Fu MC, Boddapati V, Gausden EB, et al. Surgery for a fracture of the hip within 24 hours of admission is independently associated with reduced short-term post-operative complications. Bone Joint J 2017;99-B:1216-22.

49. Surgical Efficiency Targets Program (SETP) data standardization guide. Version 8. Toronto (ON): Cancer Care Ontario; 2016.

50. Jha AK. JAMA Forum: Public reporting of surgical outcomes: surgeons, hospitals, or both? News@ JAMA 2017 Aug. 24. Available: https://newsatjama.jama.com /2017/08/24/jama-forum-public-reporting-of-surgical-outcomes-surgeons-hospitals -or-both/?linkld=41482661 (accessed 2018 May 28).

51. Wei S, Pierce O, Marshall A. Propublica Surgeon Scorecard. Available https:// projects.propublica.org/surgeons/ (accessed 2016 June 24).

52. Friedberg MW, Pronovost PJ, Shahian DM, et al. A methodological critique of the ProPublica Surgeon Scorecard. Santa Monica (CA): RAND Corporation; 2015. Available: www.rand.org/pubs/perspectives/PE170.html (accessed 2018 May 28).

53. Best practice tariff (BPT) for Fragility Hip Fracture Care User Guide. London (UK): National Hip Fracture Database; 2011. Available: www.nhfd.co.uk/20/ hipfractureR.nsf/0/9b0c5ea2e986ff56802577af0046b1df/\$FILE/Best Practice Tariff User Guide.pdf (accessed 2018 May 28).

54. Simunovic N, Devereaux PJ, Sprague S, et al. Effect of early surgery after hip fracture on mortality and complications: systematic review and meta-analysis. CMAJ 2010;182:1609-16.

55. Moja L, Piatti A, Pecoraro V, et al. Timing matters in hip fracture surgery: patients operated within 48 hours have better outcomes. a meta-analysis and meta-regression of over 190000 patients. PLoS One 2012; 7:e46175.

56. Hentschker C, Mennicken R. The volume-outcome relationship and minimum volume standards - empirical evidence for Germany. Health Econ 2015;24:644-58.

57. Resources for Optimal Care of the Injured Patient 2014 resource repository. Chicago: American College of Surgeons Committee on Trauma; 2014. Available: www.facs.org/quality programs/trauma/vrc/resources (accessed 2018 May 28).

58. Byrne JP, Nathens AB, Gomez D, et al. Timing of femoral shaft fracture fixation following major trauma: a retrospective cohort study of United States trauma centers. PLoS Med 2017;14:e1002336.

59. Redelmeier DA, Thiruchelvam D, Daneman N. Introducing a methodology for estimating duration of surgery in health services research. J Clin Epidemiol 2008;61:882-9.

60. Saltzman J, Abelson J. Overlapping surgeries to face US Senate inquiry. Boston Globe 2016 Mar. 13. Available: www.bostonglobe.com/metro/2016/03/12/senator -launches-inquiry-into-simultaneous-surgeries-hospitals-including-mass-general/ wxEsbg5r2poqtk88LkcHQl/story.html (accessed 2016 Mar. 13).

61. Mello MM, Livingston EH. Managing the risks of concurrent surgeries. JAMA 2016;315:1563-4.

\section{Competing interests: None declared.}

This article has been peer reviewed.

Affiliations: Department of Surgery (Pincus, Wasserstein, Ravi, Byrne, Nathens, Kreder, Jenkinson), University of Toronto; Institute for Clinical Evaluative Sciences (Pincus, Ravi, Paterson, Nathens, Kreder, Wodchis); Institute of Health Policy, Management and Evaluation (Pincus, Byrne, Huang, Paterson, Nathens, Kreder, Jenkinson, Wodchis), University of Toronto; Department of Surgery (Wasserstein, Ravi, Byrne, Nathens, Kreder, Jenkinson), Sunnybrook Health Sciences Centre; Toronto Rehabilitation Institute-University Health Network (Wodchis), Toronto, Ont.

Contributors: Daniel Pincus had full access to all of the data in the study and takes responsibility for the integrity of the data and the accuracy of the data analysis. Daniel Pincus, Bheeshma Ravi, David Wasserstein, J. Michael Paterson, Hans Kreder, Richard Jenkinson and Walter Wodchis were responsible for concept and design. Daniel Pincus, Bheeshma Ravi, Anjie Huang, J. Michael Paterson, Avery Nathens, Hans Kreder, Richard Jenkinson and Walter Wodchis acquired, analyzed or interpreted the data. Daniel Pincus drafted the manuscript. Daniel Pincus, James Byrne and Anjie Huang performed the statistical analysis. Daniel Pincus, Bheeshma Ravi, David Wasserstein, Anjie Huang, J. Michael Paterson, Hans Kreder, Richard Jenkinson, James Byrne and Walter Wodchis critically revised the manuscript for important intellectual content. All of the authors gave final approval of the version to be published and agreed to be accountable for all aspects of the work.

Funding: This study was funded by the Marvin Tile Chair in Orthopaedic Surgery at Sunnybrook Health Sciences Centre, Toronto, the Ontario Ministry of Health and Long-Term Care (MOHLTC) Health System Performance Research Network, and supported by the Institute for Clinical Evaluative Sciences (ICES), a nonprofit research institute funded by
MOHTLC. Daniel Pincus is supported by the Canadian Institutes of Health Research Vanier Scholarship Program.

Disclaimer: This study was supported by the Institute for Clinical Evaluative Sciences (ICES), which is funded by an annual grant from the Ontario Ministry of Health and LongTerm Care (MOHLTC). The opinions, results and conclusions reported in this paper are those of the authors and are independent from the funding sources. No endorsement by ICES or the Ontario MOHLTC is intended or should be inferred. Parts of this material are based on data and information compiled and provided by the Canadian Institute for Health Information (CIHI). However, the analyses, conclusions, opinions and statements expressed herein are those of the authors and not necessarily those of $\mathrm{CIHI}$.

Accepted: Apr. 10, 2018

Correspondence to: Daniel Pincus, d.pincus@ utoronto.ca 